\title{
Deviation from Regular Shape in the Early Stages of Formation of Strain-Driven 3D InGaAs/GaAs Micro/Nanotubes
}

\author{
Paola Frigeri, Luca Seravalli, Marco Calicchio, Enos Gombia, and Giovanna Trevisi \\ IMEM-CNR, Institute of Materials for Electronics and Magnetism, Parco Area delle Scienze 37/A, 43124 Parma, Italy \\ Correspondence should be addressed to Giovanna Trevisi; giovanna.trevisi@imem.cnr.it
}

Received 6 March 2017; Revised 17 May 2017; Accepted 4 July 2017; Published 8 August 2017

Academic Editor: Leander Tapfer

Copyright (C) 2017 Paola Frigeri et al. This is an open access article distributed under the Creative Commons Attribution License, which permits unrestricted use, distribution, and reproduction in any medium, provided the original work is properly cited.

Single-crystalline InGaAs/GaAs semiconductor micro/nanotubes have been obtained by the strain-driven self-rolling mechanism. This approach combines the advantages of bottom-up (epitaxial growth) and top-down (postgrowth processing) techniques, offering an exceptional opportunity to realize complex three-dimensional nanoarchitectures by using conventional photolithography and wet-etching processes. The method employed to obtain micro/nanotubes with selected orientation and length is described in detail. By means of high-resolution scanning electron microscopy characterization, we show a clear shape difference between single-wall and multiwalls tubes and we discuss it on the basis of strain release, taking into account also possible shape deformations induced during micro/nanotubes drying. We analyse the In-segregation profile in the nominal $\mathrm{In}_{0.20} \mathrm{Ga}_{0.80}$ As/GaAs bilayer and we show its effect on the actual diameter of the tubes, concluding that a more accurate description of the structure should consider an $\mathrm{In}_{0.20} \mathrm{Ga}_{0.80} \mathrm{As} / \mathrm{In}_{0.10} \mathrm{Ga}_{0.90} \mathrm{As} / \mathrm{GaAs}$ trilayer. This work will be useful to set up reliable methodologies for the realization of straindriven micro/nanotubes with controlled properties, necessary for their implementation in a large number of application fields.

\section{Introduction}

The miniaturization of components and devices is nowadays an essential requirement for almost all application fields. Scaling down of current technologies to obtain a real three-dimensional (3D) definition at the nanoscale is complicated and expensive, conditions that strongly limit the achievement of mass-production. Almost two decades ago, Prinz and coworkers [1] proposed a method to define micro/nanoarchitectures through the controlled release of strained membranes [2]. Since then, several authors studied and implemented this technique, highlighting the possibility of obtaining miniaturized devices without the need of complex procedures [3-5]. This technique combines bottomup and top-down methodologies. Bottom-up methods are used to create a two-dimensional (2D) system containing a certain amount of strain energy, as in the epitaxial growth of mismatched heterostructures. The top-down method consists of a photolithographic technique used to define a pattern on the structure. The strain of the system can be released by removing a sacrificial layer using wet-etching procedures.
This results in a shape transition of the freestanding $2 \mathrm{D}$ system into a 3D structure.

Several 3D shapes have been reported [4] pointing out the unique feature of this approach: the combination of nanoscale dimensions given by the epitaxial growth and microscale size defined by lithography. One of the most studied 3D shape is based on tube formation as a result of strain-driven rolling-up of 2D membranes [3]. These micro/nanotubes ( $\mu$ tubes) have been proposed for several applications: tubular-based photonic integrated components (filters, modulators, directional couplers, and lasers) obtained by embedding active layers such as quantum dots and quantum wells in the heterostructure [6], novel sensors directly integrated into microfluidic channels (lab-in-a-tube) [7], and also energy-storage microdevices [8].

A deeper understanding of the relationship between structural parameters of the starting $2 \mathrm{D}$ system and the properties of strain-driven 3D structures is clearly needed to implement such applications. To this aim, we focus on the study of $\mu$ tubes formed starting from $\mathrm{InGaAs} / \mathrm{GaAs}$ bilayers grown by Molecular Beam Epitaxy (MBE). MBE 


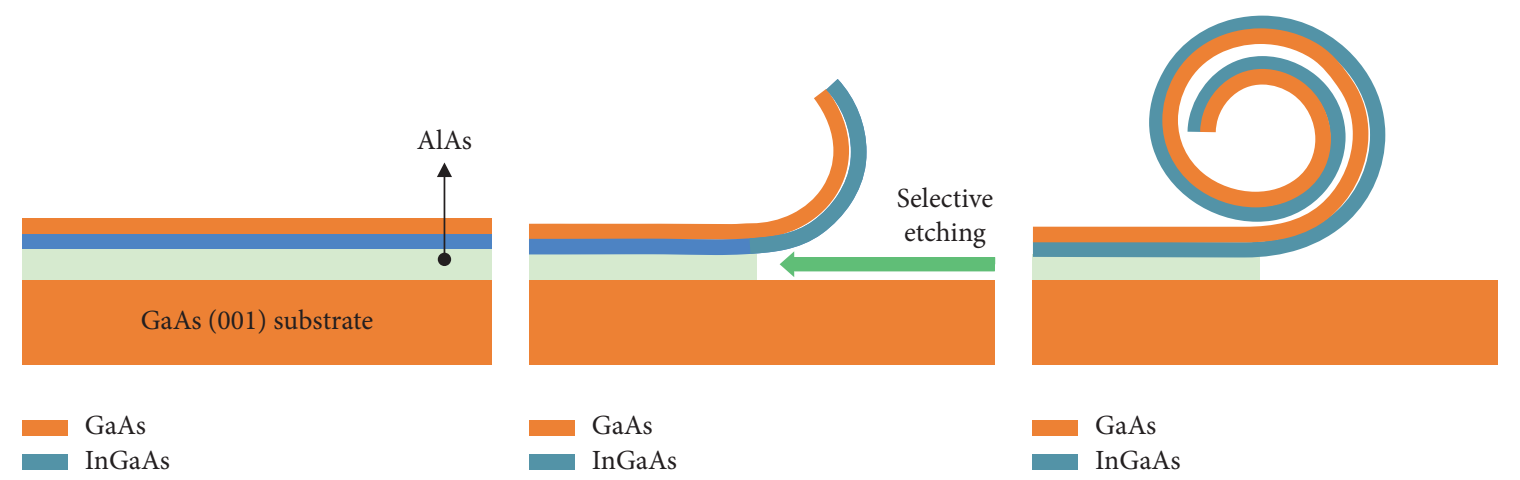

(a)

(b)

(c)

FIGURE 1: Schematic representation of the $\mu$ tube fabrication process: (a) as-grown epitaxial structure; (b) the InGaAs/GaAs bilayer is free to bend as a result of the selective etching of the underlying AlAs sacrificial layer; and (c) $\mu$ tube formation.

is a technique that allows a high degree of control on the deposition of heterostructures and, then, is particularly suited for this work [9]. The semiconductor structure is patterned using conventional optical lithography and the bilayer is released by a wet-etching. This last step allows the study of the very first stages of the rolling-up process which, as we observe, shows deviations from the ideal rolled-up $\mu$ tube. The actual structure of the bilayer is investigated through the comparison of photoluminescence (PL) results and energy levels calculation of the as-grown heterostructure, based on structure parameters derived by X-ray diffraction (XRD). The results of this study indicate the presence of an In-segregation profile, typical of In-containing MBEgrown structures [10]. Due to this effect, we conclude that the actual structure should be better described by an $\mathrm{In}_{0.20} \mathrm{Ga}_{0.80} \mathrm{As} / \mathrm{In}_{0.10} \mathrm{Ga}_{0.90} \mathrm{As} / \mathrm{GaAs}$ trilayer, rather than the nominal $\mathrm{In}_{0.20} \mathrm{Ga}_{0.80} \mathrm{As} / \mathrm{GaAs}$ bilayer. Using the trilayer parameters, we calculate the $\mu$ tube diameter and we find a substantial agreement with the experimental diameter measured by scanning electron microscopy (SEM).

Our study highlights the relevance of 2D structure deposition procedures and parameters to control the features of the final 3D architectures. This is of interest to correctly design rolled-up micro/nanostructures with desired properties for direct implementation into practical devices.

\section{Materials and Methods}

The growth of the InGaAs/GaAs bilayer structure was carried out in a solid-source Varian Gen II MBE system, on (100)oriented GaAs semi-insulating substrate (see Figure 1(a)). After the desorption of the native oxide from the GaAs substrate surface, a $5 \mathrm{~nm}$ thick AlAs buffer layer was grown at $600^{\circ} \mathrm{C}$; the thickness of AlAs was chosen to optimize the postgrowth processing of the structure [11]. The substrate temperature was then lowered to $500^{\circ} \mathrm{C}$ during a growth interruption of $60 \mathrm{~s}$ under Arsenic flux to grow the InGaAs/GaAs bilayer. This lower temperature is instrumental to minimize Indium-segregation and desorption during InGaAs deposition and alloy intermixing at InGaAs-GaAs interface. The InGaAs thickness is well below the critical value predicted by the Matthews-Blakeslee model for the formation of misfit dislocations [12]. The evolution of the reflection high-energy electron diffraction (RHEED) pattern during MBE deposition indicated that the InGaAs was pseudomorphic to GaAs, thus assuring a high crystal quality of the structure. The strain state of InGaAs/GaAs bilayer was confirmed by double crystal XRD measurements in conjunction with detailed rocking curve analysis. By XRD analysis we were able to obtain a reliable estimation of the thicknesses and compositions of layers in the structure [13], as discussed in Results and Discussion.

Continuous-wave photoluminescence (PL) was performed at $10 \mathrm{~K}$ on the as-grown structure using the $532 \mathrm{~nm}$ line of a DPSS laser as excitation, with a maximum power density of about $5 \mathrm{~W} / \mathrm{cm}^{2}$. The PL spectra were measured by a Fast-Fourier Transform spectrometer with a $1 \mathrm{meV}$ resolution and a cooled Ge detector.

$\mathrm{XRD}$ values of thicknesses and compositions were used to calculate the quantum confined energy levels of the planar structure (Figure 1(a)), in order to compare the expected emission energies with PL characterization. Calculation of the quantum system composed by the AlAs, InGaAs, and GaAs layers was performed by means of the software Tibercad, following the procedure described in depth in [14]. We considered a conduction-band offset $Q c=\Delta E c / \Delta E v$ of 0.52 (inclusive of strain effects) and a value of the exciton binding energies of $10 \mathrm{meV}$ [15].

The design parameters of the epitaxial bilayer determine the $\mu$ tube diameters and the $\mu$ tube nanowall thicknesses, since these features are controlled by the value of the lattice mismatch and by the relative thickness of InGaAs and GaAs layers. On the contrary, the final shape and the positioning of $3 \mathrm{D}$ microstructures on the substrate are controlled by the photolithographic patterning. To define the pattern, we used conventional photolithography and two consecutive wetchemical etching processes with different etching solutions.

First, on the surface of epitaxial structure we prepared a positive photoresist micropattern consisting of an array of rectangular shapes with the same width $(40 \mu \mathrm{m})$ and different lengths, ranging from 50 to $3200 \mu \mathrm{m}$ (50, 100, $200,400,800,1600$, and $3200 \mu \mathrm{m}$ ). The edges of rectangles 


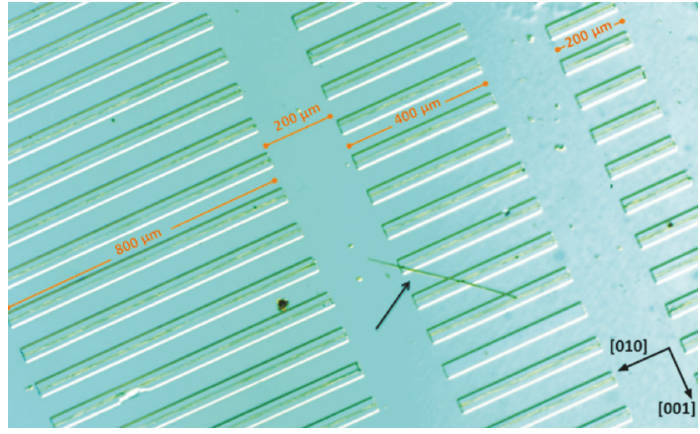

Figure 2: Optical image of an array of InGaAs/GaAs rolled-up microstructures obtained after a $t=45 \mathrm{~s}$ etching. The photolithography pattern consists of rectangular mesas with length ranging from 200 to $800 \mu \mathrm{m}$ and width of $40 \mu \mathrm{m}$, the orientation of the mesas in shown in the image. The arrow indicates a 3D microstructure completely detached from the substrate. Full scale image $(460 \times$ 308) $\mu \mathrm{m}$.

were precisely aligned along the $\langle 001\rangle$ directions that are known to be the preferred rolling directions on (100) GaAs, as a consequence of Young's modulus anisotropy in cubic semiconductor crystals $[16,17]$.

Then, we performed the first wet-chemical etching $\left(\mathrm{H}_{2} \mathrm{SO}_{4}: \mathrm{H}_{2} \mathrm{O}_{2}: \mathrm{H}_{2} \mathrm{O}=1: 8: 80\right.$ for $\left.12 \mathrm{~s}\right)$ to transfer the geometric pattern down to the AlAs sacrificial buffer layer. The etched profiles obtained were investigated by Atomic Force Microscopy (AFM). We measured a uniform etching depth of $60 \pm 5 \mathrm{~nm}$, deep enough to uncover the AlAs surrounding the rectangular mesa structures.

Finally as shown in Figures 1(b) and 1(c), to release the bilayer from the substrate, AlAs sacrificial layer was underetched in a high selective diluted $\mathrm{HF}$ solution $\left(\mathrm{HF}: \mathrm{H}_{2} \mathrm{O}\right.$ $=1: 2$ ) [18]. The addition of a surfactant (benzalkonium chloride) and the gentle stirring of HF solution during the etching process were required to optimize the etching efficiency and to improve the spatial homogeneity of the $3 \mathrm{D}$ InGaAs/GaAs $\mu$ tubes [19]. Both the wet-chemical etchings were performed at room temperature. The structures were rinsed in two steps: first in deionized (DI) water for 5 minutes and then in methanol for the following 5 minutes. The structures were dried on a hot-plate for $10 \mathrm{~s}$ at $70^{\circ} \mathrm{C}$. The use of a low surface tension solvent $(0.0227 \mathrm{~N} / \mathrm{m}$ for methanol against $0.0728 \mathrm{~N} / \mathrm{m}$ for water, both at $25^{\circ} \mathrm{C}$ ) allows preventing the tubes from collapsing during the evaporation of the rinsing solution [20, 21]. In addition, we followed the work by Seleznev et al. [22] to calculate the collapsing pressure $\Delta P$ and the critical pressure $P_{\mathrm{cr}}$ (see (1) and (2) of [22], resp.) relative to our structures. When $\Delta P$ exceeds $P_{\text {cr }}$, the tubes are expected to collapse. Considering methanol as rinsing liquid, we obtain $\Delta P=0.54$ bar and $P_{\mathrm{cr}}=1.39 \mathrm{bar}$, thus showing that our structures are not expected to collapse.

An optical microscope image of the $\mu$ tube array obtained by longer etching time ( $45 \mathrm{~s}$ ) is shown in Figure 2.

Field-Emission SEM was used to investigate the morphology of the $\mu$ tubes in $54^{\circ}$-tilted configurations. The $54^{\circ}$-tilted images are automatically corrected for vertical parallax to

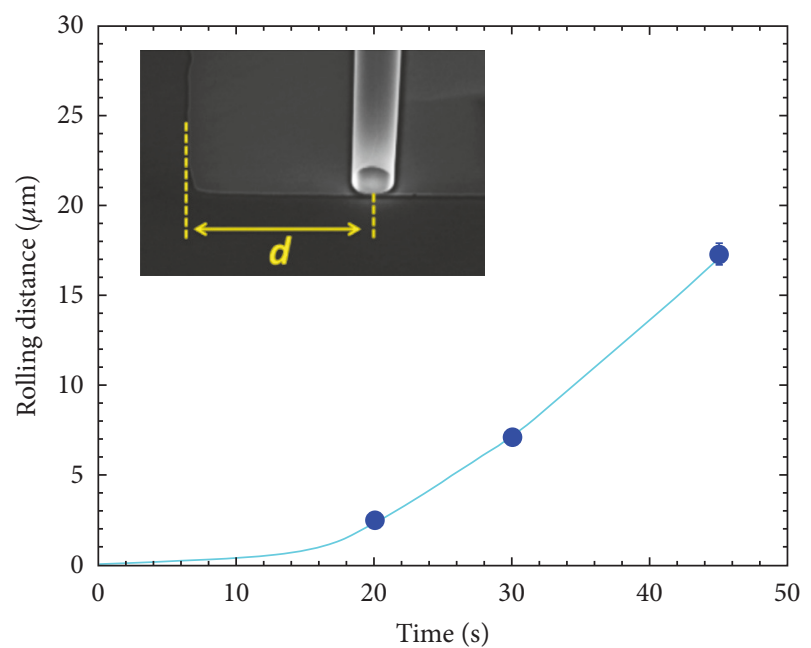

FIgURE 3: Rolling distance $(d)$ as a function of the etching time $(t)$; $d$ has been measured from SEM images (see the inset) on different $\mu$ tubes giving error bars almost comparable to the spot size.

allow length measurements along the growth direction. SEM images were obtained with $5 \mathrm{keV}$ acceleration voltage of the electron beam and $5 \mathrm{~mm}$ working distance.

\section{Results and Discussion}

As shown by Chun et al. $[23,24], \mu$ tubes grown on GaAs (001) substrates preferentially roll-up along the $\langle 001\rangle$ directions. Additionally, the actual rolling direction of $\mu$ tubes is determined by geometrical conditions. Given a rectangular mesa shape oriented along the $\langle 001\rangle$ directions, experimental results and theoretical calculations demonstrate that longside rolling is energetically favoured. This is shown in the optical microscope image of Figure 2, where $\mu$ tubes are oriented along the [010] direction corresponding to the longer side of the rectangular mesas.

In order to study the features of the rolling process, we analysed the shape of $\mu$ tubes as a function of the etching time $(t)$ of the underlying sacrificial layer. By increasing $t$, a larger fraction of the InGaAs/GaAs bilayer detaches from the underlying substrate and is free to bend. In Figure 3 we plot the rolling distances $(d)$ experimentally measured from several SEM images of the $\mu$ tubes as functions of the etching times. Assuming that $d=0$ when $t=0$, from the plot we extrapolate a very low value for the initial etching velocity $\left(v_{e}\right)$. This may stem from the existence of an oxidized AlAs layer that constitutes a barrier for the etching. As soon as the oxide is etched away, the bilayer starts detaching with a $v_{e}$ $=0.68 \mu \mathrm{m} / \mathrm{s}$ constant velocity proving that the procedure we chose grants a uniform etching of the sacrificial layer.

By reducing $t$ to a few tens of seconds, we can analyse the very first stages of rolling-up. With reference to Figure 4, we distinguish three regimes:

(i) $t=20 \mathrm{~s}, d=2.5 \pm 0.18 \mu \mathrm{m}$ : this is the initial stage of rolling-up where the InGaAs/GaAs freestanding layer starts bending (Figure 4(a)); 


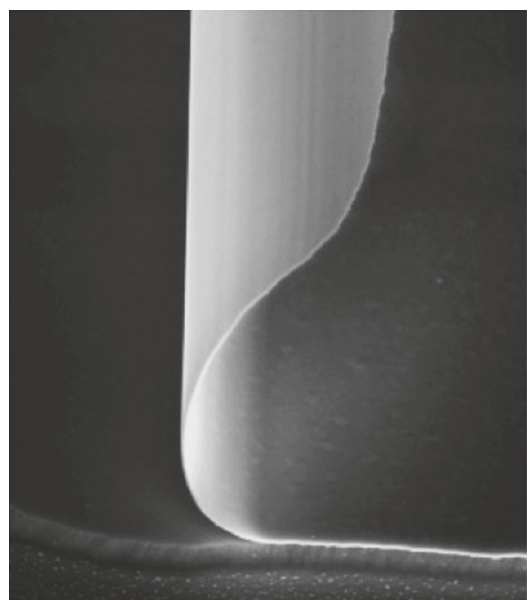

(a)

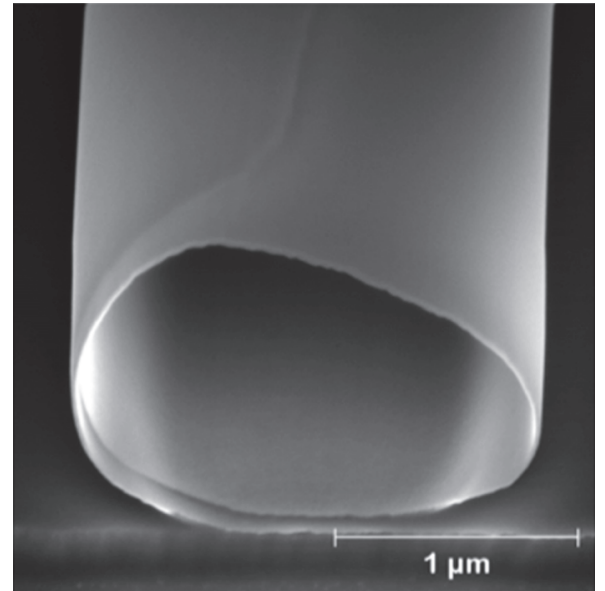

(b)

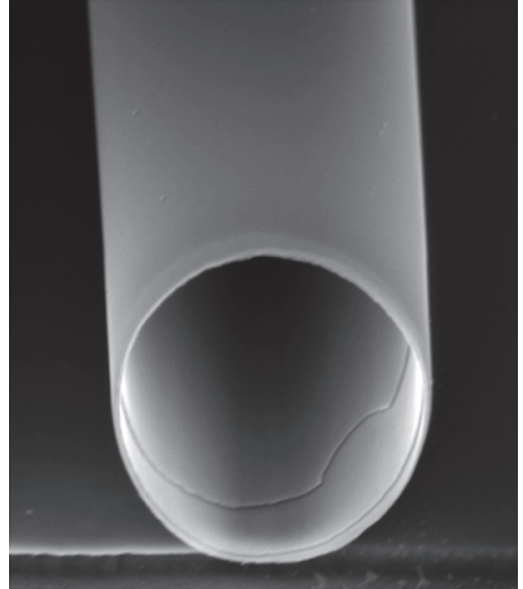

(c)

FIGURE 4: High-resolution SEM images of $\mu$ tubes obtained with different etching times: (a) $t=20 \mathrm{~s}$, (b) $t=30 \mathrm{~s}$; and (c) $t=45 \mathrm{~s}$. The scale bar is the same for all the images.

(ii) $t=30 \mathrm{~s}, d=7.13 \pm 0.35 \mu \mathrm{m}$ : the bilayer rolls up into the tube. SEM magnification shows an irregular shape of the tube section (Figure 4(b));

(iii) $t=45 \mathrm{~s}, d=17.3 \pm 0.6 \mu \mathrm{m}$ : this stage corresponds to the formation of $\mu$ tubes with several foldings. SEM image shows a perfectly circular shape of the tube section (Figure 4(c)).

SEM images in Figure 4 show that only the $\mu$ tubes obtained with a $45 \mathrm{~s}$ etching possess the expected circular sections while the structures resulting from shorter etching times show an irregular shape. Usually, shape deformations in single-wall $\mu$ tubes are attributed to partial or complete collapse during the drying process [22]. In the previous section we showed that our specific conditions for $\mu$ tube drying prevent the collapse of $\mu$ tubes. Anyhow, the model proposed by Seleznev et al. [22] does not take into account capillary forces between the substrate and the walls that could induce an additional shape deformation. Our experimental study allows studying and comparing the very first stages of the rolling-up process and gives the possibility of accurately analysing different shape effects. While nonhomogeneous curvature radius of the bilayer in the proximity of the substrate can be influenced by capillary forces, Figures 4(a) and 4 (b) show a change of curvature radius also in the proximity of the $\mu$ tube free edge. A reason for this behaviour could be that an additional lateral strain is released in the proximity of the free edge of the bilayer, while this is not possible in the inner region of the freestanding bilayer. As a result, the curvature radius becomes larger as the distance from the edge decreases, as indeed observed in Figure 4(a). Figure 4(b), corresponding to a complete $\mu$ tube, still maintains an irregular shape. As clearly visible from the SEM image, a strong curvature change of the $\mu$ tube section takes place when the outer folding overlaps with the underlying free edge of the bilayer. By further increasing the number of foldings (Figure 4(c)), the shape becomes regular with a homogeneous curvature radius. We explain this behaviour due to the balance between different curvature radii of the overlapping bilayer: when the number of foldings increases, the free edge is flattened and is forced to assume the curvature of the inner regions of the bilayer. The dependence of the lattice parameter of the tubes on the proximity to the edge bound to the substrate has been shown by Krause et al. [25] through X-ray microdiffraction analysis of several types of tubes. This experimental evidence supports the idea of the existence of regions of the freestanding bilayer subjected to different strain.

On the $t=45 \mathrm{~s} \mu$ tube (Figure 4(c)) we measured an internal diameter of $1.582 \mu \mathrm{m}$. Following the work by Deneke et al. [19] the diameter of rolled-up $\mu$ tubes can be related to the thickness and composition of the materials that constitute the bilayer. As is known, the epitaxial growth of In-containing compounds on GaAs is affected by segregation, a phenomenon that may strongly change the effective composition profile of the structure [10]. Through X-ray measurements and calculations of the energy-band structure, we already showed that PL emission of buried $\operatorname{In}(\mathrm{Ga}) \mathrm{As} / \mathrm{GaAs}$ quantum wells is strongly dependent on their effective In-content profile [15]. To take into account such effects, we analysed the PL emission of the as-grown structure and modeled the band structure in order to compare the experimental and calculated emission energies; these study is performed starting from X-ray measured thicknesses and compositions (Figure 5). As a result of the segregation, the nominal $\mathrm{In}_{0.2} \mathrm{Ga}_{0.8}$ As $(5 \mathrm{~nm}) / \mathrm{GaAs}(11.5 \mathrm{~nm})$ bilayer turns out to be better described by an $\operatorname{In}_{0.2} \mathrm{Ga}_{0.8}$ As $(3.3 \mathrm{~nm}) / \mathrm{In}_{0.1} \mathrm{Ga}_{0.9}$ As $(4 \mathrm{~nm}) / \mathrm{GaAs}(11.5 \mathrm{~nm})$ trilayer. As a further confirmation, the thickness of the trilayer structure $(18.8 \mathrm{~nm})$ well compares to the thickness of the $\mu$ tube walls measured from highmagnification SEM images $(18.7 \pm 1 \mathrm{~nm})$, as shown in Figure 6.

In order to calculate the expected value of $\mu$ tube diameter by using the actual parameters of the structure, we followed 


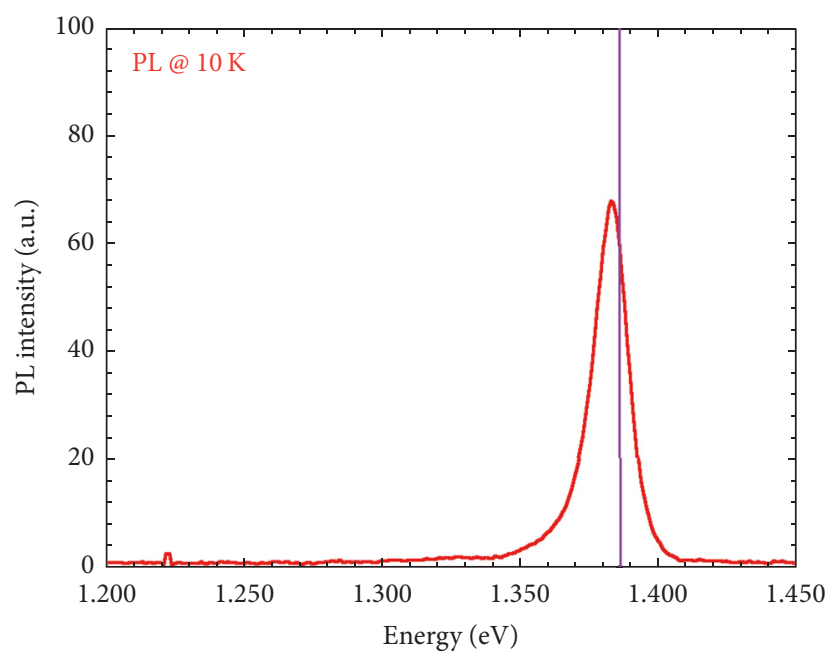

FiguRE 5: PL spectrum of the as-grown structure measured at $10 \mathrm{~K}$ with peak emission energy at $1.383 \mathrm{eV}$ (red line) compared to the calculated emission energy of the structure with X-ray derived parameters, $1.387 \mathrm{eV}$ (purple line).

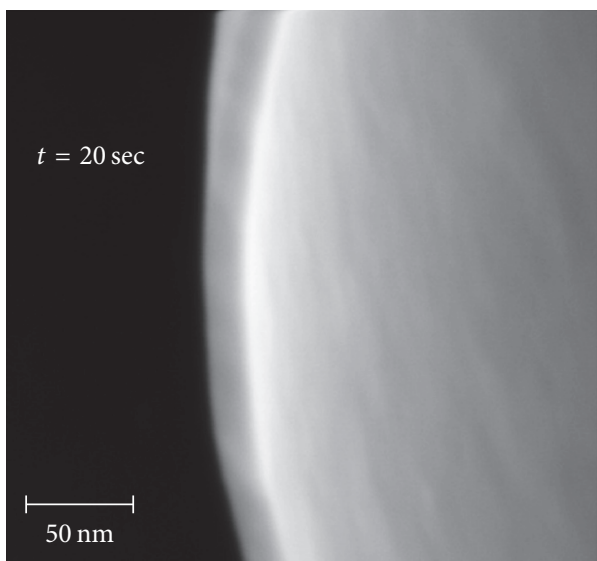

(a)

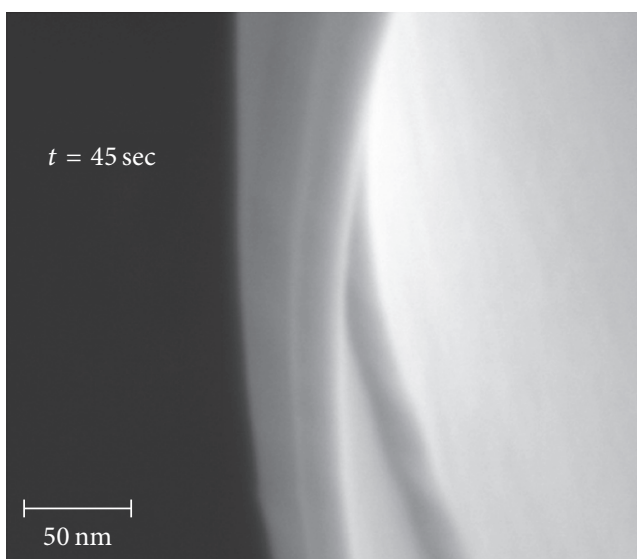

(b)

FIGURE 6: High-resolution and high-magnification SEM images of the $\mu$ tube nanowalls for structures with (a) 1 folding and (b) 3 foldings. The experimentally measured thickness of the nanowalls is $18.7 \pm 1 \mathrm{~nm}$.

the approach proposed by Nishidate and Nikishkov in [26] for the calculation of generalized plane strain deformation of multilayer structures. On the basis of plane strain elasticity theory, authors derive curvature estimation for strained multilayer structure with an initial strain due to lattice mismatch. In our study the strained structure consists of three layers of thicknesses $t_{1}=3.3 \mathrm{~nm}, t_{2}=4 \mathrm{~nm}$ and $t_{3}=11.5 \mathrm{~nm}$. The curvature of a trilayer can be calculated by setting $n=3$ in (13) of [26], where $y_{i}$ values are defined as $y_{0}=0, y_{1}=t_{1}$, $y_{2}=t_{1}+t_{2}$, and $y_{3}=t_{1}+t_{2}+t_{3}$ and $\varepsilon_{1}{ }^{0}=\left(a_{1}-a_{\mathrm{GaAs}}\right) / a_{\mathrm{GaAs}}$, $\varepsilon_{2}{ }^{0}=\left(a_{1}-a_{2}\right) / a_{\mathrm{GaAs}}$, and $\varepsilon_{3}{ }^{0}=0$, with $a_{1}, a_{2}$, and $a_{\mathrm{GaAs}}$ given by the lattice constants of $\operatorname{In}_{0.2} \mathrm{Ga}_{0.8} \mathrm{As}, \mathrm{In}_{0.1} \mathrm{Ga}_{0.9} \mathrm{As}$, and $\mathrm{GaAs}$, respectively. Following this notation, $\varepsilon_{1}{ }^{0}, \varepsilon_{2}{ }^{0}$, and $\varepsilon_{3}{ }^{0}$ are the initial strains in the lower- $\operatorname{In}_{0.2} \mathrm{Ga}_{0.8} \mathrm{As}$, middle$\mathrm{In}_{0.1} \mathrm{Ga}_{0.9}$ As, and upper-GaAs layer. Exact values of Young modulus and Poisson ratio for the different materials were considered to solve the generalized multilayer plane strain equation using Matlab. A value of the $\mu$ tube diameter of
$1.764 \mu \mathrm{m}$ was obtained, against the experimental value of $1.62 \pm 0.08 \mu \mathrm{m}$ obtained from the statistical analysis of $\mu$ tubes as the one shown in Figure $4(\mathrm{c})$. The discrepancy between theoretical and experimental values could be due to additional strains on the structure surface and/or to the consumption of the top GaAs oxide during wet-chemical process in HF solution, as suggested by Deneke et al. [19]. The typical thickness of GaAs oxide in these structures is in the order of a few monolayers (ML) [27]. By taking into account a GaAs thickness reduction of $4 \mathrm{ML}$, we calculated a diameter of $1.608 \mu \mathrm{m}$ in excellent agreement with the experimental value.

\section{Conclusions}

Here we have presented the fabrication of InGaAs/GaAs $\mu$ tubes with nanometer-sized walls, thanks to the straininduced self-rolling of MBE-grown bilayers. Lengths and 
location of $\mu$ tubes on the substrate are controlled by mesa structures defined by conventional photolithographic processing, while micro/nanotube diameters depend on the lattice mismatch and on the thickness of the InGaAs/GaAs structure.

We have discussed in detail all the technological steps needed to obtain such 3D microstructures: (i) MBE growth of starting structure, consisting of layers of AlAs, GaAs, and pseudomorphic InGaAs, (ii) XRD and PL characterization of the epitaxial structure, (iii) photolithography, and (iv) wetchemical etching.

SEM characterization of rolled-up structures has allowed us to understand the mechanisms of the self-rolling process. In particular, we observe an evident difference between single-wall and multiwalls tubes that we ascribe in part to capillary forces between the substrate and the $\mu$ tube walls but also to a different strain status of the rolled-up bilayer. When the outer edge of the bilayer is free to elastically relax an increase in the curvature radius is observed in comparison with the situation when the edge is constrained to bend under other foldings of the bilayer.

Structural parameters of the epitaxial layer were obtained by XRD characterization and validated by comparing PL emission energies with model calculations of quantum levels related to the AlAs/InGaAs/GaAs quantum well. We showed that the structure is actually an $\mathrm{In}_{0.2} \mathrm{Ga}_{0.8} \mathrm{As} /$ $\mathrm{In}_{0.1} \mathrm{Ga}_{0.9} \mathrm{As} / \mathrm{GaAs}$ trilayer, rather than an $\operatorname{In}_{0.2} \mathrm{Ga}_{0.8} \mathrm{As} / \mathrm{GaAs}$ bilayer. This picture is confirmed by SEM high-magnification of walls and by theoretical calculation of curvature radius.

A trilayer is expected to have rather different properties than a bilayer such as band profiles, strain status, and refraction index mismatch with air. All of these are crucial parameters for applications of nanotubes as nanoelectronic elements, as systems for strain engineering of embedded nanostructures (such as quantum dots), as optical and chemical sensors.

Hence, we believe that the results we have shown here will contribute to a deeper understanding of the properties of these $3 \mathrm{D}$ nanostructures.

\section{Conflicts of Interest}

The authors declare that there are no conflicts of interest regarding the publication of this paper.

\section{Acknowledgments}

This work has been partially supported by Fondazione Monte Parma. The authors acknowledge E. Buffagni, F. Rossi, and C. Ferrari for XRD characterization.

\section{References}

[1] V. Y. Prinz, V. A. Seleznev, A. K. Gutakovsky et al., "Freestanding and overgrown InGaAs/GaAs nanotubes, nanohelices and their arrays," Physica E, vol. 6, no. 1, pp. 828-831, 2000.

[2] O. G. Schmidt and K. Eberl, "Thin solid films roll up into nanotubes," Nature, vol. 410, no. 6825, article 168, 2001.
[3] S. Böttner, M. R. Jorgensen, and O. G. Schmidt, "Rolled-up nanotechnology: 3D photonic materials by design," Scripta Materialia, vol. 122, pp. 119-124, 2016.

[4] Z. Chen, G. Huang, I. Trase, X. Han, and Y. Mei, "Mechanical self-assembly of a strain-engineered flexible layer: wrinkling, rolling, and twisting," Physical Review Applied, vol. 5, no. 1, article 017001, 2016.

[5] X. Li, "Strain induced semiconductor nanotubes: from formation process to device applications," Journal of Physics D: Applied Physics, vol. 41, no. 19, Article ID 193001, 2008.

[6] S. Mendach, S. Kiravittaya, A. Rastelli, M. Benyoucef, R. Songmuang, and O. G. Schmidt, "Bidirectional wavelength tuning of individual semiconductor quantum dots in a flexible rolled-up microtube," Physical Review B, vol. 78, no. 3, article 035317, 2008.

[7] E. J. Smith, W. Xi, D. Makarov et al., "Lab-in-a-tube: ultracompact components for on-chip capture and detection of individual micro-/nanoorganisms," Lab on a Chip, vol. 12, no. 11, pp. 1917-1931, 2012.

[8] J. Deng, X. Lu, L. Liu, L. Zhang, and O. G. Schmidt, "Introducing rolled-up nanotechnology for advanced energy storage devices," Advanced Energy Materials, vol. 6, no. 23, article 1600797, 2016.

[9] P. Frigeri, L. Seravalli, G. Trevisi, and S. Franchi, "Molecular beam epitaxy: an overview," in Reference Module in Materials Science and Materials Engineering, S. Hashmi, Ed., pp. 1-40, Elsevier, Oxford, UK, 2016.

[10] K. Muraki, S. Fukatsu, Y. Shiraki, and R. Ito, "Surface segregation of in atoms during molecular beam epitaxy and its influence on the energy levels in InGaAs/GaAs quantum wells," Applied Physics Letters, vol. 61, no. 5, pp. 557-559, 1992.

[11] K. S. Koteswara Rao, T. Katayama, S. Yokoyama, and M. Hirose, "Optimum atomic spacing for alas etching in gaas epitaxial liftoff technology," Japanese Journal of Applied Physics, vol. 39, pp. L457-L459.

[12] J. W. Matthews and A. E. Blakeslee, "Defects in epitaxial multilayers. I. Misfit dislocations," Journal of Crystal Growth, vol. 27, no. C, pp. 118-125, 1974.

[13] Y. C. Chen, P. K. Bhattacharya, and J. Singh, "Accurate determination of misfit strain, layer thickness, and critical layer thickness in ultrathin buried strained InGaAs/GaAs layer by Xray diffraction," Journal Of Vacuum Science \& Technology B, vol. 10, no. 2, pp. 769-771, 1992.

[14] L. Seravalli, C. Bocchi, G. Trevisi, and P. Frigeri, "Properties of wetting layer states in low density InAs quantum dot nanostructures emitting at $1.3 \mu \mathrm{m}$ : effects of InGaAs capping," Journal of Applied Physics, vol. 108, no. 11, article 114313, 2010.

[15] L. Seravalli, G. Trevisi, P. Frigeri, F. Rossi, E. Buffagni, and C. Ferrari, "Wetting layer states in low density InAs/InGaAs quantum dots from sub-critical InAs coverages," Journal of Physics D: Applied Physics, vol. 46, no. 31, article 315101, 2013.

[16] W. A. Brantley, "Calculated elastic constants for stress problems associated with semiconductor devices," Journal of Applied Physics, vol. 44, no. 1, pp. 534-535, 1973.

[17] I. S. Chun and X. Li, "Controlled assembly and dispersion of strain-induced InGaAs/GaAs nanotubes," IEEE Transactions on Nanotechnology, vol. 7, no. 4, pp. 493-495, 2008.

[18] E. Yablonovitch, T. Gmitter, J. P. Harbison, and R. Bhat, "Extreme selectivity in the lift-off of epitaxial GaAs films," Applied Physics Letters, vol. 51, no. 26, pp. 2222-2224, 1987.

[19] C. Deneke, C. Müller, N. Y. Jin-Phillipp, and O. G. Schmidt, "Diameter scalability of rolled-up In(Ga)As/GaAs nanotubes," Semiconductor Science and Technology, vol. 17, no. 12, pp. 12781281, 2002. 
[20] I. S. Chun, Strain-induced self-rolled-up semiconductor micro/ nanotubes: fabrication and characterization, [Ph.D. thesis], URL http, 2011, http://hdl.handle.net/2142/29778.

[21] M. T. Todaro, L. Blasi, C. Giordano et al., "Nanowalled polymer microtubes fabricated by using strained semiconductor templates," Nanotechnology, vol. 21, no. 24, article 245305, pp. 1-5, 2010.

[22] V. Seleznev, H. Yamaguchi, Y. Hirayama, and V. Prinz, "Singleturn GaAs/InAs nanotubes fabricated using the supercritical $\mathrm{CO}_{2}$ drying technique," Japanese Journal of Applied Physics, vol. 42, no. 7A, pp. L791-L794, 2003.

[23] I. S. Chun, V. B. Verma, V. C. Elarde et al., "InGaAs/GaAs 3D architecture formation by strain-induced self-rolling with lithographically defined rectangular stripe arrays," Journal of Crystal Growth, vol. 310, no. 7-9, pp. 2353-2358, 2008.

[24] I. S. Chun, A. Challa, B. Derickson, K. J. Hsia, and X. Li, "Geometry effect on the strain-induced self-rolling of semiconductor membranes," Nano Letters, vol. 10, no. 10, pp. 3927-3932, 2010.

[25] B. Krause, C. Mocuta, T. H. Metzger, C. Deneke, and O. G. Schmidt, "Local structure of a rolled-up single crystal: an X-ray microdiffraction study of individual semiconductor nanotubes," Physical Review Letters, vol. 96, no. 16, article 165502, 2006.

[26] Y. Nishidate and G. P. Nikishkov, "Generalized plane strain deformation of multilayer structures with initial strains," Journal of Applied Physics, vol. 100, no. 11, article 113518, 2006.

[27] C. Deneke, N.-Y. Jin-Phillipp, I. Loa, and O. G. Schmidt, "Radial superlattices and single nanoreactors," Applied Physics Letters, vol. 84, no. 22, pp. 4475-4477, 2004. 

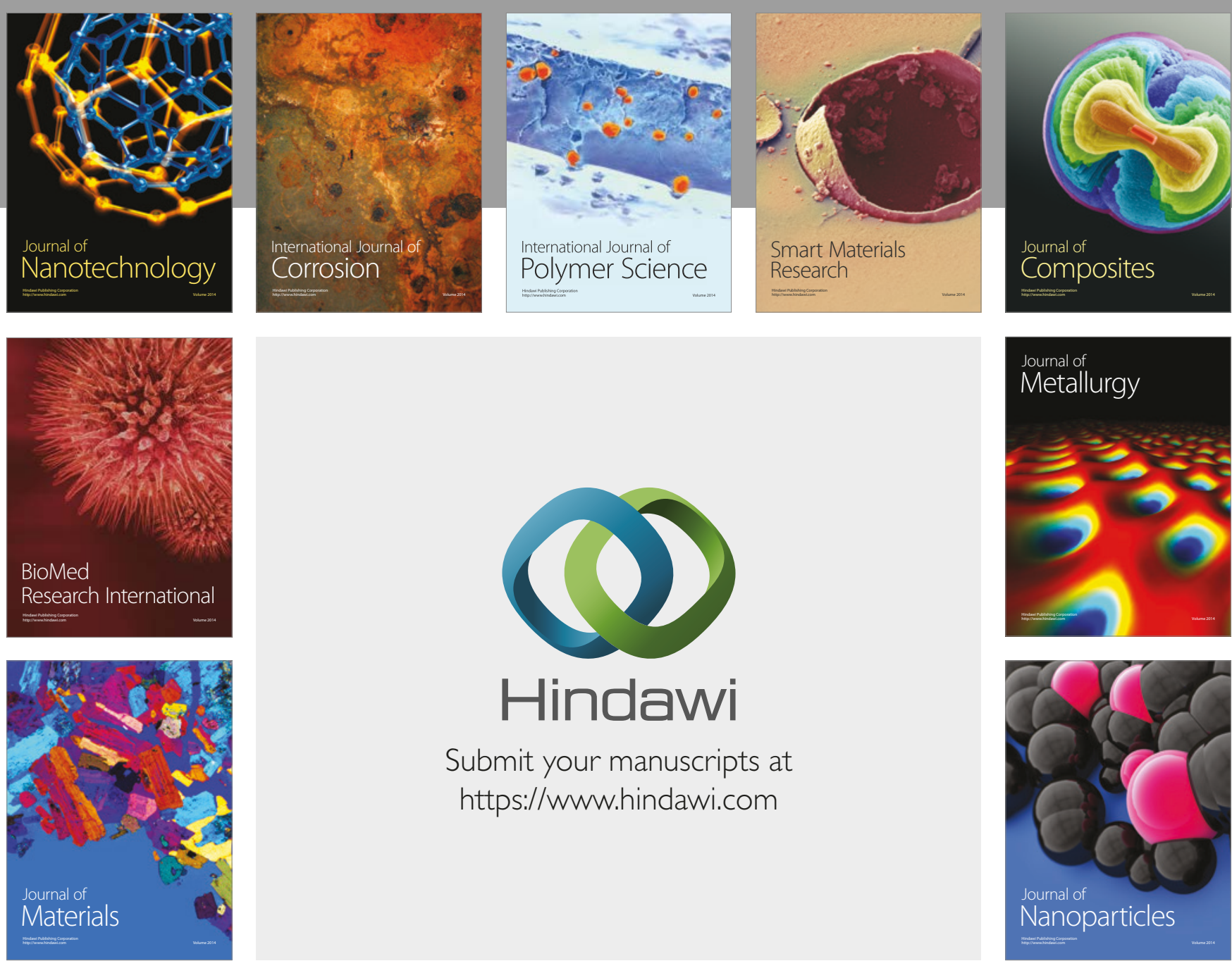

\section{Hindawi}

Submit your manuscripts at

https://www.hindawi.com
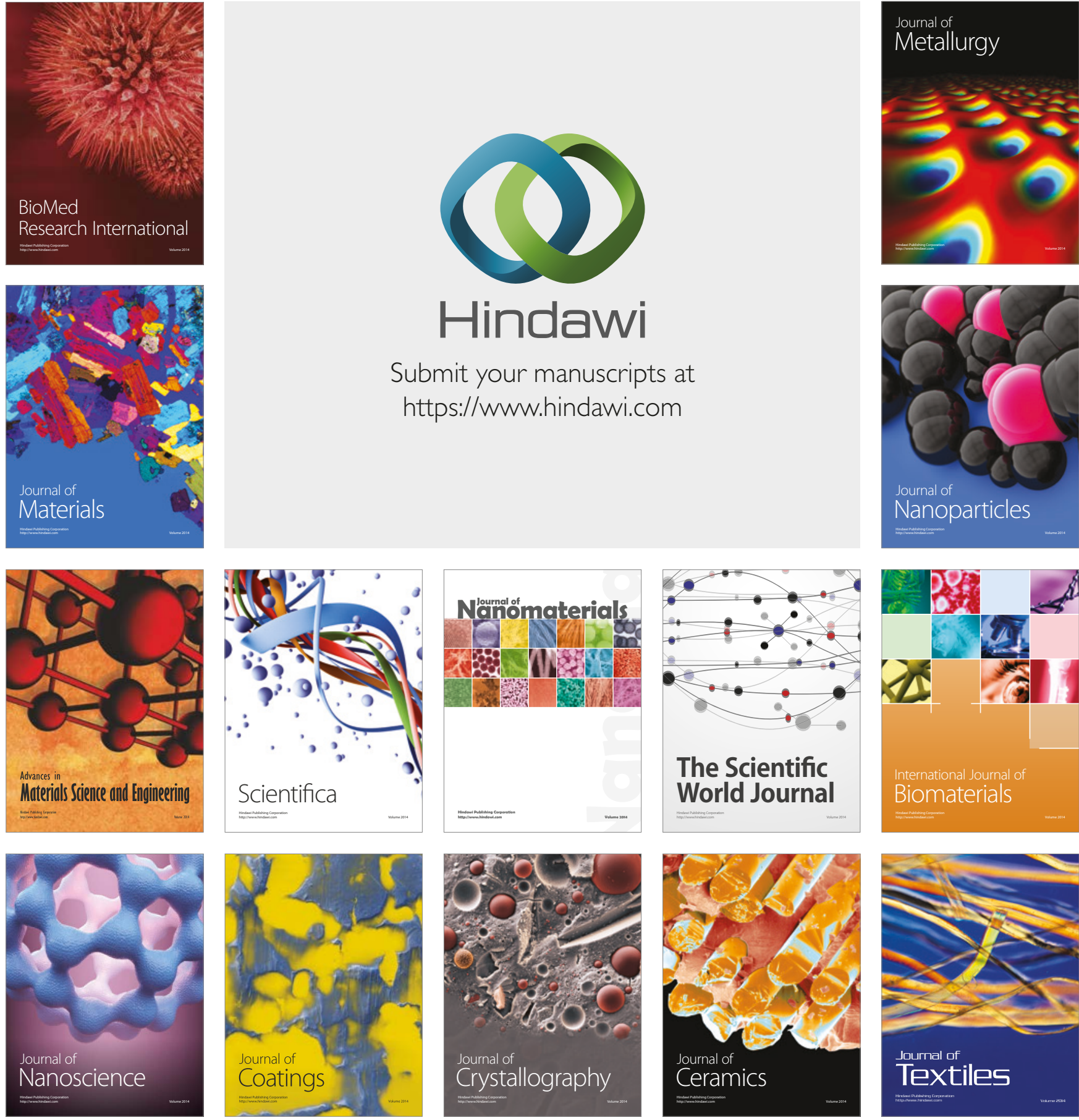

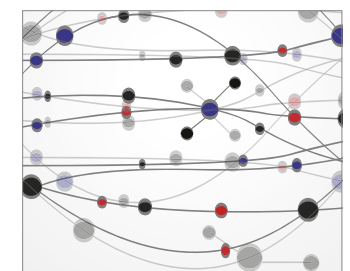

The Scientific World Journal
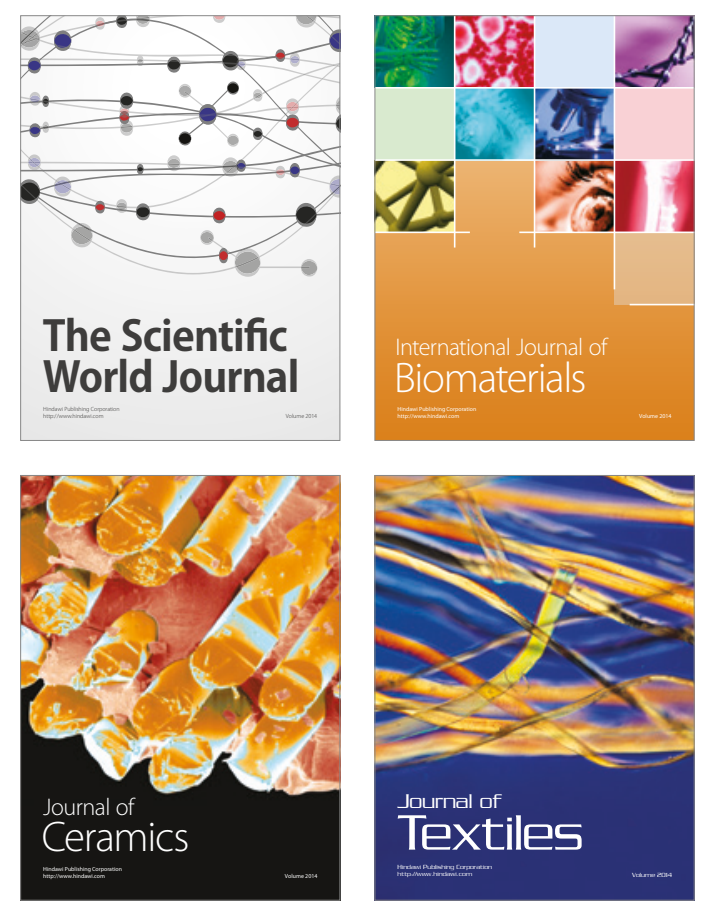